\title{
The effect of a low carbohydrate high fat diet on emerging biochemical markers of cardiometabolic risk
}

\author{
Deaglan McCullough ${ }^{1,2}$, Tanja Harrison ${ }^{1}$, Katie Lane ${ }^{1}$, Lynne Boddy ${ }^{2}$, \\ Farzad Amirabdollahian ${ }^{3}$, Michael Schmidt ${ }^{4}$, Kevin Enright ${ }^{1}$, Claire Stewart ${ }^{2}$ and Ian Davies ${ }^{1}$ \\ ${ }^{1}$ School of Sport Studies, Leisure and Nutrition, Liverpool John Moores University, Liverpool, United Kingdom, \\ ${ }^{2}$ School of Sports and Exercise Sciences, Liverpool John Moores University, Liverpool, United Kingdom, \\ ${ }^{3}$ School of Health Sciences, Liverpool Hope University, Liverpool, United Kingdom and \\ ${ }^{4}$ Advanced Pattern Analysis and Countermeasures Group, Research Innovation Centre, Colorado state University, \\ Fort Collins, USA
}

\begin{abstract}
Worldwide, cardiovascular disease (CVD) is the number 1 cause of mortality and is associated with insulin resistance (IR). Emerging biomarkers such as FGF21 and adiponectin are associated with cardiometabolic risk. Low carbohydrate, high fat (LCHF) diets have been reported to reduce cardiometabolic risk markers; however, few studies have compared a LCHF diet vs. a high carbohydrate (HC), lower fat diet under ad libitum conditions on adiponectin and FGF21. The purpose of this study was to investigate the effects of an ad libitum LCHF vs. HC diet on IR, FGF21 and adiponectin in 16 healthy adults. Ethical approval: Liverpool John Moores University Research Ethics Committee (16/ELS/029); registered with ClinicalTrials.gov (Ref. NCT03257085). Participants were randomly assigned to a HC diet ( $\mathrm{n}=8$, the UK Eatwell guidelines; $\geq 50 \%$ of energy from carbohydrates) or a LCHF diet $(\mathrm{n}=8$, consume $<50 \mathrm{~g} / \mathrm{day}$ of carbohydrates). All provided plasma samples at 0, 4 and 8 weeks. FGF21 (R\&D Systems) was analysed via ELISA and adiponectin, insulin and glucose were analysed via immunoassay technology (Randox Evidence Investigator ${ }^{\mathrm{TM}}$ Metabolic Syndrome Arrays I \& II). Mann Whitney, Friedmans, Wilcoxon tests and $2 \times 3$ ANOVA (IBM SPSS $25^{\circledR}$ ) were undertaken to investigate significant differences between and within groups. The homeostatic model assessment (HOMA) was used to calculate IR. FGF21 significantly $(P=0.04)$ decreased (Mdn, IQR:148.16, 78.51-282.02 to 99.4, 39.87-132.29 pg/ml) after 4 weeks and significantly $(P=0.02)$ increased $(M d n$, IQR:167.38, 80.82-232.89 pg/ml) by 8 weeks vs. baseline with LCHF. No significant differences $(P>0.05)$ were observed between groups. Adiponectin was significantly $(P=0.03)$ different at week 4 only between groups. Adiponectin increased after 4 weeks ( $M d n, I Q R: 13.44,9.12-25.47$ to $16.64,11.96-21.51 \mathrm{ng} / \mathrm{ml})$ but was only significantly $(P=0.03)$ different by 8 weeks vs. baseline in the HC group $(M d n, I Q R: 16,10.8-27.43 \mathrm{ng} / \mathrm{ml})$. Adiponectin remained unchanged $(P=0.96)$ in the LCHF group. HOMA significantly decreased with both diets after 8 weeks only (mean \pm SD, LCHF: $2.9 \pm 1.3$ to $1.8 \pm 0.8$, HC: $2.5 \pm 0.6$ to $1.9 \pm 0.6, P=0.008$ ) but was not significantly $(P=0.60)$ different between groups. These preliminary data reveal that while both diets improved insulin sensitivity, they may do so by different mechanisms. Future studies are warranted to investigate further, how a LCHF vs. HC diet affects FGF21 and adiponectin, and the subsequent regulation of IR. Furthermore, studies that extend these findings by determining the impact of LCHF vs. HC on peripheral metabolism to determine potential nutrition-mediated mechanisms of metabolic adaptation are warranted.
\end{abstract}

\section{Conflict of Interest}

There is no conflict of interest. 